\section{Cell-bound complement activation products associate with lupus severity in SLE}

To cite: Arriens C, Alexander RV, Narain S, et al. Cell-bound complement activation products associate with lupus severity in SLE. Lupus Science \& Medicine 2020;7:e000377. doi:10.1136/ lupus-2019-000377

- Additional material is published online only. To view please visit the journal online (http://dx.doi.org/10.1136/ lupus-2019-000377).

These data were presented at the American College of Rheumatology in 2017.

Received 21 December 2019 Revised 10 April 2020 Accepted 17 April 2020

\section{Check for updates}

(C) Author(s) (or their employer(s)) 2020. Re-use permitted under CC BY-NC. No commercial re-use. See rights and permissions. Published by BMJ.

For numbered affiliations see end of article.

Correspondence to Dr Roberta Vezza Alexander; ralexander@exagen.com

\section{ABSTRACT}

Objectives To evaluate the association between lupus severity and cell-bound complement activation products (CB-CAPs) or low complement proteins C3 and C4.

Methods All subjects $(n=495)$ fulfilled the American College of Rheumatology (ACR) classification criteria for SLE. Abnormal CB-CAPs (erythrocyte-bound C4d or Blymphocyte-bound C4d levels >99th percentile of healthy) and complement proteins C3 and C4 were determined using flow cytometry and turbidimetry, respectively. Lupus severity was estimated using the Lupus Severity Index (LSI). Statistical analysis consisted of multivariable linear regression and groups comparisons.

Results Abnormal CB-CAPs were more prevalent than low complement values irrespective of LSI levels $(62 \%$ vs $38 \%$, respectively, $\mathrm{p}<0.0001$ ). LSI was low (median 5.44, IQR: 4.77-6.93) in patients with no complement abnormality, intermediate in patients with abnormal CB-CAPs (median 6.09, IQR: 5.31-8.20) and high in the group presenting with both abnormal CB-CAPs and low C3 and/or C4 (median 7.85, IQR: 5.51-8.37). Odds of immunosuppressant use was higher in subjects with LSI $\geq 5.95$ compared with subjects with LSI $<5.95$ ( 1.60 vs $0.53, p<0.0001$ for both). Multivariable regression analysis revealed that higher LSI scores associated with abnormal CB-CAPs—but not low C3/C4-after adjusting for younger age, race and longer disease duration $(p=0.0001)$, which were also independent predictors of disease severity (global $R^{2}=0.145$ ).

Conclusion Abnormalities in complement activation as measured by CB-CAPs are associated with increased LSI.

\section{INTRODUCTION}

SLE is a complex, chronic autoimmune disease characterised by autoantibody production and immune system dysregulation resulting in multiorgan inflammation and potentially damage.

Low complement levels, commonly found in patients with SLE, generally indicate complement activation, although they could represent decreased synthesis. Typical complement assessments include serum determinations of C3, C4 and in some cases CH50. Low complement is not included in the American College of Rheumatology (ACR) 1997 classification criteria ${ }^{1}$; however, it is a component of the criteria put forth by the Systemic Lupus Erythematosus International Collaborating Clinics ${ }^{2}$ and the European League Against Rheumatism and $\mathrm{ACR}^{3}$ because it is an important feature of dysregulated immunity in SLE. ${ }^{4}$

We and others have shown previously that complement activation can be measured by the accumulation of C4d on the surface of haematopoietic cells, including erythrocytebound C4d (EC4d) and B-lymphocyte-bound C4d (BC4d). These biomarkers, collectively known as cell-bound complement activation products (CB-CAPs), ${ }^{5}$ have superior diagnostic accuracy for lupus ${ }^{6} 7$ compared with low serum complement. ${ }^{8}$ Beyond aiding in diagnosing SLE, CB-CAPs are useful for monitoring SLE disease activity as prognostic biomarkers, although their utility as predictive biomarkers awaits further study. ${ }^{9}$

Variability in disease manifestations and severity between different patients as well as within patients over their disease course is common. Although an instrument such as the Safety of Estrogen in Lupus National Assessment - Systemic Lupus Erythematosus Disease Activity Index (SELENA-SLEDAI) reflects disease activity around the time of clinical evaluation, it does not measure cumulative disease burden. As cumulative disease severity can lead to organ damage, instruments that measure disease severity are important to identify subjects at risk of major organ involvement. The Lupus Severity Index (LSI) was 
developed as a tool for scoring a subject's disease severity based on weighting of ACR classification criteria derived from one's treatment history. ${ }^{10}$ The elements necessary to score the LSI do not require an in-person examination and are easily available in medical records and research datasets. Thus, the LSI can be calculated retrospectively for large subject cohorts.

In this cross-sectional study of patients with SLE, we correlated standard complement (C3 and C4) and CB-CAPs with LSI scores.

\section{METHODS}

\section{Study population}

This study reports a cross-sectional subanalysis of studies enrolling adult subjects with SLE $(\mathrm{n}=495)$ at 17 academic centres. Central or internal review boards approved the studies and subjects provided informed consent.

All subjects were diagnosed with SLE and fulfilled the 1997 ACR $^{1}$ classification criteria. Medication regimens were available for 438 subjects; 209 were on one or more immunosuppressants, including methotrexate $(n=40)$, azathioprine $(n=53)$, mycophenolate $(n=105)$, belimumab $(n=12)$, rituximab $(n=2)$, oral ciclosporin $(n=2)$, cyclophosphamide $(n=3)$ or intravenous immunoglobulins $(n=1)$.

Venous blood samples were tested at Exagen in our clinical laboratory accredited by the College of American Pathologists.

\section{Lupus severity index}

LSI was determined by weighting and summation of ACR criteria and subcriteria as previously described ${ }^{10}$ (see online supplementary material).

\section{Biomarker analysis}

Serum C3 and C4 were determined by immunoturbidimetry (The Binding Site, San Diego, California, USA) ${ }^{11}$ and were considered low if below the manufacturer's lower limits of normal $(81.1$ and $12.9 \mathrm{mg} / \mathrm{dL}$ for C3 and C4, respectively). Low complement status refers to low C3 and/or low C4.

Complement activation was determined using CB-CAPs measured by quantitative flow cytometry. ${ }^{11}$ CB-CAPs were considered abnormal if levels of EC4d and/or BC4d were above the 99th percentile of a group of healthy individuals $(>14$ and $>60$ net mean fluorescence intensity, respectively) ${ }^{7811}$ Abnormal CB-CAPs status refers to abnormal EC4d and/or abnormal BC4d.

Anti-double-stranded DNA (dsDNA) antibodies were determined by ELISA (Quanta Lite, Inova Diagnostics, San Diego, California, USA). All serum samples above $301 \mathrm{IU} / \mathrm{mL}$ were further tested by indirect immunofluorescence assay (IFA) using the Crithidia luciliae assay (Nova-Lite, Inova Diagnostics). Anti-dsDNA antibodies were considered positive if confirmed by IFA.

\section{Statistical analysis}

Multivariable linear regression analysis was used to model the relative contributions of low complement and elevated CB-CAPs to LSI with race, gender, age and disease duration as covariates. Regression analysis of a subset of patients included medication and renal disease activity as additional covariates. McNemar's test was used to compare CB-CAPs to standard complement testing. Fisher's exact test, analysis of variance and Kruskal-Wallis tests were used for group comparisons as appropriate. Odds of immunosuppressant use were evaluated by binomial distribution analysis.

\section{RESULTS}

The demographic characteristics of the 495 subjects included in this study are reported in table 1.

Overall, per cent positivity was $62 \%$ for CB-CAPs and $38 \%$ for low complement $(\mathrm{p}<0.0001)$. Anti-dsDNA was positive in approximately a third of the population (table 1).

Median LSI was 5.95 (IQR (5.20-8.17)) and scores ranged from 3.27 to $9.38($ mean $\pm \mathrm{SD}=6.48 \pm 1.6)$. The majority of subjects were females $(91 \%)$ and presented with LSI slightly lower (median 5.9 (5.2-8.2)) compared with males (median 7.5 (5.3-8.3)). LSI was highest in Asian subjects (7.1 (5.4-8.3)), followed by AfricanAmerican/black (6.7 (5.4-8.2)), Latino/Hispanic (6.6 $(5.4-8.2))$, other races $(5.8(5.4-8.0))$, and lowest in the Caucasian/white subjects $(5.6(4.9-8.0))(\mathrm{p}<0.001)$.

Among the 495 subjects, 153 had both low complement and abnormal CB-CAPs, 153 had abnormal CB-CAPs alone, 37 had low complement alone and 152 presented with no complement abnormalities (normal complement and normal CB-CAPs). LSI score was highest in the double positive group, intermediate in the subjects with low complement or abnormal CB-CAPs only and lowest in those with neither abnormality $(\mathrm{p}<0.001)$ (table 1$)$.

As the LSI distribution across the entire patient population showed two peaks, similar to findings by Bello $e t a l^{10}$ (online supplementary figure 1), subjects were divided into two groups based on LSI: 247 subjects had low LSI (LSI <5.95, median 5.21 (4.66-5.49)) and 248 had high LSI (LSI $\geq 5.95$, median 8.17 (7.54-8.51)). Both low complement and abnormal CB-CAPs were more prevalent in the high LSI group; interestingly, abnormal CB-CAPs was more prevalent than low complement in both groups $(\mathrm{p}<0.0001$ for both) (figure 1).

Binomial distribution analysis showed that the odds of immunosuppressant use in subjects with LSI $\geq 5.95$ was 1.60 while it was 0.53 in the subjects with LSI $<5.95$ ( $\mathrm{p}<0.0001$ for both).

Univariate analysis results revealed that younger age at visit, younger age at diagnosis, low complement (C3 and/ or $\mathrm{C} 4$ ), race, positive anti-dsDNA, use of corticosteroids or immunosuppressants and abnormal CB-CAPs (EC4d and/or BC4d) associated with higher LSI (table 2, top). Multivariable linear regression analysis revealed abnormal CB-CAPs, younger age at visit, longer disease duration, and race as the strongest predictors of current LSI (table 2, bottom); low complement (estimate $=0.279 \pm 0.152$, 


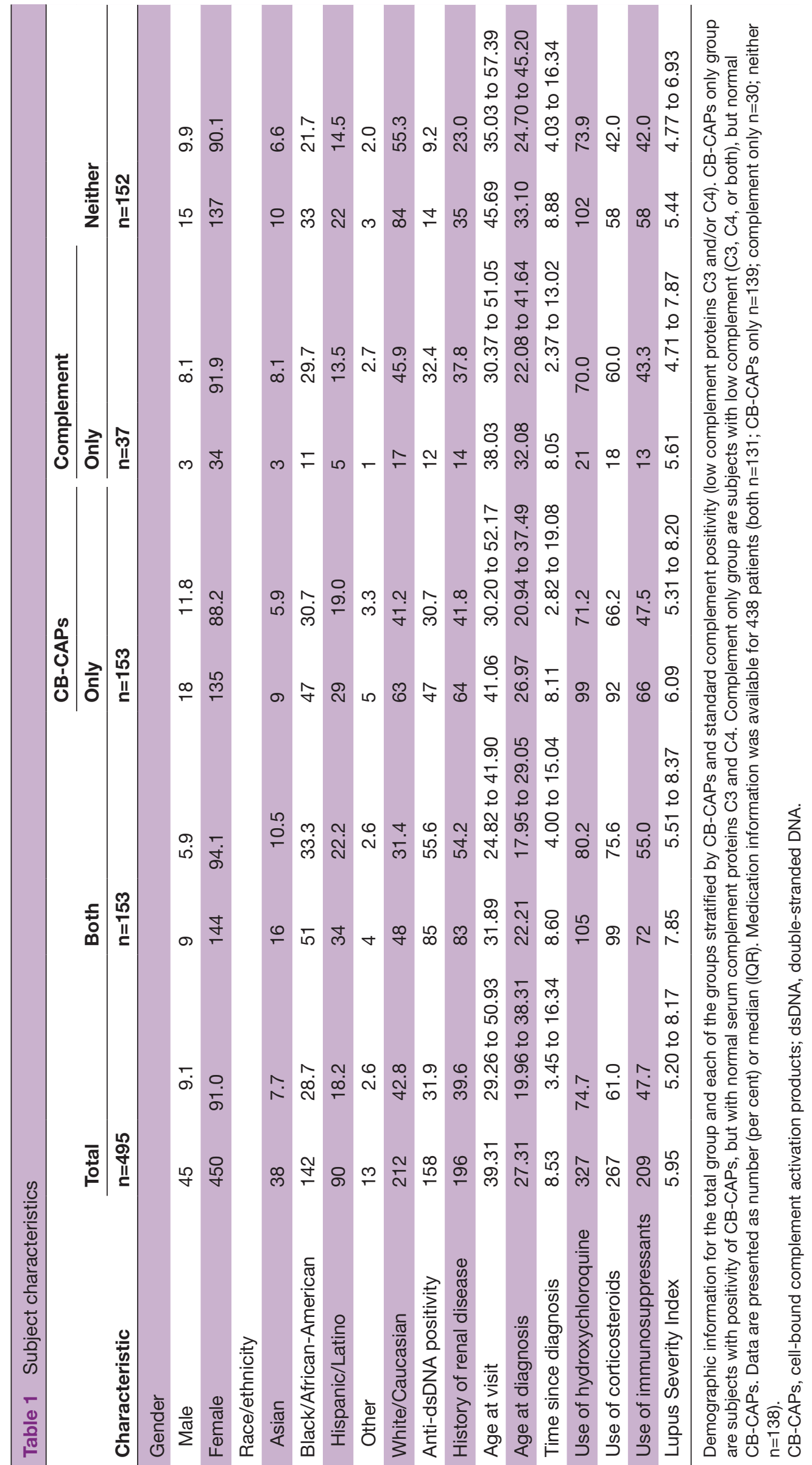




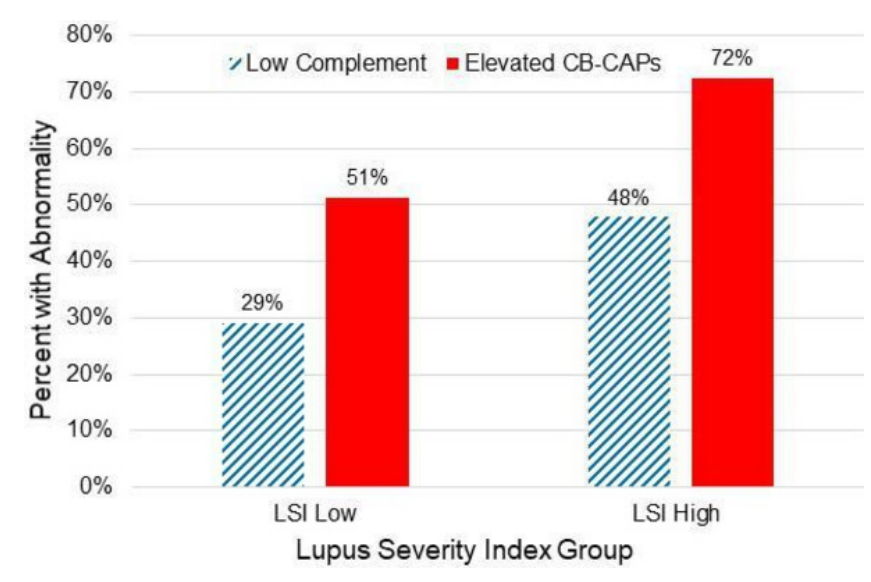

Figure 1 Comparison of low complement and elevated cellbound complement activation products (CB-CAPs) by Lupus Severity Index (LSI) group. Percentage of subjects with low complement (low serum complement proteins C3 and/or C4) and elevated CB-CAPs (elevated EC4d and/or BC4d) by LSI groups.

$\mathrm{p}=0.098)$ and gender (estimate $=0.438 \pm 0.240, \mathrm{p}=0.068$ ) were not significantly associated with the LSI and, therefore, were not included in the model (see online supplementary table 1 for an additional analysis that included low complement and gender and online supplementary table 5 for an additional analysis that included positive anti-dsDNA, low complement and gender). Overall, the model accounted for $14.5 \%$ of the total variability of the LSI, and abnormal CB-CAPs alone remained a significant predictor of current LSI (estimate $=0.697 \pm 0.148$, $\mathrm{p}<0.0001)$ after adjusting for age at visit, time since diagnosis and race.

Analysis of a subset of patients for whom medication information was available $(n=438)$ found that use of immunosuppressants correlated with higher LSI (online supplementary table 2) when adjusting for covariates of CB-CAPs, race, gender, age and disease duration (parameter estimate $0.727, \mathrm{p}<0.0001$ ). In a subset of patients for whom SELENA-SLEDAI renal activity information was available $(\mathrm{n}=446)$, renal activity correlated with LSI (parameter estimate 1.937, $\mathrm{p}<0.0001$ ) after adjusting for age, disease duration and CB-CAPs (online supplementary table 3 ). In patients for whom both medication and renal activity information was available $(n=402)$, CB-CAPs remained significant predictors of LSI (parameter estimate $0.760, p<0.0001)$ after controlling for immunosuppressant use, renal activity and age at visit (online supplementary table 4). Additionally, we show the progressive effect on the CB-CAPs' significance as predictors of LSI as additional variables are added to the final parsimonious model, and the resulting $\mathrm{R}^{2}$ of each model are detailed in online supplementary table 6 .

\section{DISCUSSION}

Complement activation has a central role in SLE. ${ }^{5}$ In this study, we evaluated the contribution of complement activation and, more specifically, of the classical complement pathway, on SLE severity using the LSI.

In our cross-sectional, multicentre study of 495 wellcharacterised lupus subjects, we found that complement abnormalities (both low C3/C4 and abnormal CB-CAPs) were more prevalent in subjects with more severe disease based on their LSI score. When subjects were stratified by LSI into low and high groups, a higher percentage of individuals in both groups were CB-CAPs positive compared with having low complement. This is consistent with the higher sensitivity of CB-CAPs compared with low complement in SLE observed in this and other studies ${ }^{6-8}$ and indicates that complement activation as measured by CB-CAPs reflects disease severity more accurately than low C3/C4. Consistent with these data, although low standard complement C3/C4 associated with elevated LSI, the association was no longer significant after adjusting for age and race. However, CB-CAPs correlated with LSI and remained significant after adjusting for race, age and time since diagnosis. LSI was highest in Asian subjects, followed by African-American/black subjects, Latino/ Hispanic subjects, other and lowest in the Caucasian/ white subjects. These racial differences in LSI are in agreement with previous data ${ }^{10}$ and with the fact that SLE is more aggressive in non-white individuals. ${ }^{12}$

Younger age at diagnosis and younger age at visit were associated with higher LSI, as well as longer time between diagnosis and visit. Due to collinearity between age at diagnosis and age at visit, age at diagnosis was not included as a covariate.

Racial and age-related factors are known to have an impact on lupus severity. ${ }^{12-14}$ We now show that abnormalities in the complement system and, in particular, classical complement activation as measured by CB-CAPs, are associated with increased LSI.

As the LSI was derived from immunosuppressant use as a proxy for disease severity, ${ }^{10}$ it is not surprising that the odds of immunosuppressant use was high in the subjects with more severe disease (LSI $\geq 5.95$ ). In addition, the analysis of a subset of patients with available medication information showed that use of immunosuppressants correlated (but was not collinear) with LSI scores as determined by stepwise multivariable analysis.

LSI also correlated with SELENA-SLEDAI renal activity in a subset analysis. Though these data were not available for all patients in our dataset, these additional analyses demonstrated that CB-CAPs remained significant predictors of LSI when adjusting for significant covariates.

The cross-sectional evaluation of a time-expanded concept like the LSI is a limitation of this study. In addition, disease severity in SLE can be influenced by multiple factors, while the LSI is based only on weighted ACR criteria and subcriteria. In the original validation study, the LSI predicted early mortality, however, it may not capture all the elements that contribute to disease severity in SLE ${ }^{10}$ Calculation of the Katz Lupus Severity of Disease index, ${ }^{15}$ which reflects disease damage, was not 
Table 2 Top: Association of LSI with low complement, elevated CB-CAPs, race/ethnicity, gender, age, age at diagnosis and disease duration (time since diagnosis) as determined by univariate analysis. These variables, except age at diagnosis due to collinearity with age at visit, were included in a stepwise model building analysis. Bottom: Association of LSI with elevated CBCAPs, race/ethnicity, age and disease duration (time since diagnosis), as determined by stepwise multivariable analysis. The model found that gender and low complement (low C3 and/or C4) were not significant when controlling for the other variables $\left(p=0.068\right.$ and $p=0.098$, respectively); therefore, they were not included in the final model $\left(R^{2}=0.145\right)$

Top: Univariate analysis

\begin{tabular}{|c|c|c|c|c|}
\hline Factor & $\begin{array}{l}\text { Parameter } \\
\text { estimate }\end{array}$ & SE & $95 \% \mathrm{Cl}$ & $P$ value \\
\hline \multicolumn{5}{|l|}{ Gender } \\
\hline Female & Ref & & & \\
\hline \multicolumn{5}{|l|}{ Race/ethnicity } \\
\hline Black/African-American & 0.660 & 0.175 & 0.316 to 1.003 & 0.0002 \\
\hline Hispanic/Latino & 0.600 & 0.203 & 0.201 to 0.999 & 0.0033 \\
\hline Other & 0.602 & 0.461 & -0.303 to 1.508 & 0.1920 \\
\hline White/Caucasian & Ref & & & \\
\hline Age at visit & -0.025 & 0.005 & -0.035 to -0.015 & $<0.0001$ \\
\hline Elevated CB-CAPs & 0.958 & 0.145 & 0.672 to 1.244 & $<0.0001$ \\
\hline Anti-dsDNA positivity & 0.548 & 0.156 & 0.241 to 0.855 & 0.0005 \\
\hline Use of hydroxychloroquine & -0.221 & 0.180 & -0.575 to 0.134 & 0.2221 \\
\hline Use of corticosteroids & 0.641 & 0.158 & 0.330 to 0.952 & $<0.0001$ \\
\hline Use of immunosuppressants & 0.893 & 0.151 & 0.595 to 1.190 & $<0.0001$ \\
\hline
\end{tabular}

CB-CAPs, cell-bound complement activation products; dsDNA, double-stranded DNA; LSI, Lupus Severity Index.

\section{Bottom: Final multivariable model}

\begin{tabular}{|c|c|c|c|c|}
\hline \multirow[b]{2}{*}{ Factor } & \multirow[t]{2}{*}{$\begin{array}{l}\text { Parameter } \\
\text { estimate }\end{array}$} & \multirow[t]{2}{*}{ SE } & \multirow[b]{2}{*}{$95 \% \mathrm{Cl}$} & \multirow[b]{2}{*}{$P$ value } \\
\hline & & & & \\
\hline \multicolumn{5}{|l|}{ Race/ethnicity } \\
\hline Asian & 0.646 & 0.272 & 0.112 to 1.179 & 0.0177 \\
\hline Black/African-American & 0.466 & 0.173 & 0.127 to 0.805 & 0.0071 \\
\hline Other & 0.435 & 0.441 & -0.432 to 1.301 & 0.3246 \\
\hline White/Caucasian & Ref & & & \\
\hline Age at visit & -0.024 & 0.006 & -0.035 to -0.013 & $<0.0001$ \\
\hline Time since diagnosis & 0.033 & 0.008 & 0.016 to 0.049 & 0.0001 \\
\hline Elevated CB-CAPs & 0.697 & 0.148 & 0.406 to 0.987 & $<0.0001$ \\
\hline
\end{tabular}

possible in this study as some of the elements of this index were not collected for all patients in the dataset.

The findings of this study expand on our previous work that shows association of CB-CAPs and, in particular, EC4d, with SLE disease activity. ${ }^{9}$ Taken together, our data suggest that complement activation as measured by CB-CAPs parallels disease activity and associates with disease severity, especially in younger or non-white subjects and in those with long-standing disease. However, data need to be interpreted with caution as the model accounted for a small fraction $(14.5 \%)$ of the total variability of the LSI. 
Evaluation of the ability of CB-CAPs to predict future lupus severity through inception and longitudinal studies will be important to the field.

\section{Author affiliations}

${ }^{1}$ Oklahoma Medical Research Foundation, Oklahoma City, Oklahoma, USA

${ }^{2}$ University of Oklahoma Health Sciences Center, Oklahoma City, Oklahoma, USA

${ }^{3}$ Exagen Inc, Vista, California, USA

${ }^{4}$ Rheumatology, Northwell Health, Great Neck, New York, USA

${ }^{5}$ Center for Musculoskeletal Care, New York University, New York, New York, USA

${ }^{6}$ MedStar Washington Hospital Center, Washington, District of Columbia, USA

${ }^{7}$ Attune Health, Beverly Hills, California, USA

${ }^{8}$ Rheumatology, Harvard University, Boston, Massachusetts, USA

${ }^{9}$ Rheumatology, University iof California San Diego, La Jolla, California, USA

${ }^{10}$ Division of Rheumatology, Albert Einstein College of Medicine, Bronx, New York, USA

${ }^{11}$ Azrieli Faculty of Medicine, Bar llan University, Zefat, Israel

${ }^{12}$ Research Institute, Galilee Medical Center, Nahariya, Israel

${ }^{13}$ Medicine/Rheumatology, Northwestern University, Chicago, Illinois, USA

${ }^{14}$ New York University School of Medicine, New York, New York, USA

${ }^{15}$ Rheumatology, Columbia University Medical Center, New York, New York, USA

${ }^{16}$ Allegheny Health Network, Pittsburgh, Pennsylvania, USA

Acknowledgements We thank Armida Sace, Rowena LaFon and JoAnne Ligayon for technical assistance in biomarker analysis and Claudia Ibarra for the management and supervision of the clinical laboratory.

Contributors Study design: CA, RVA and TD. Data analysis: CA, RVA, TD and JC. Authorship and editing of manuscript: CA and RVA.

Funding This work was funded by Exagen.

Competing interests RVA, JC, TO and TD are current or former employees of Exagen. AW is a consultant to Exagen and chief medical officer. CA, SN, AS, CEC, DJW, EM, KCK, CP, RR-G, JPB, AA, RF, SM, JA have received research support from Exagen.

Patient consent for publication Not required.

Ethics approval Internal review board approved the study and informed consent was collected for all subjects.

Provenance and peer review Not commissioned; externally peer reviewed.

Open access This is an open access article distributed in accordance with the Creative Commons Attribution Non Commercial (CC BY-NC 4.0) license, which permits others to distribute, remix, adapt, build upon this work non-commercially, and license their derivative works on different terms, provided the original work is properly cited, appropriate credit is given, any changes made indicated, and the use is non-commercial. See: http://creativecommons.org/licenses/by-nc/4.0/.

\section{ORCID iDs}

Cristina Arriens http://orcid.org/0000-0002-9546-1664
Roberta Vezza Alexander http://orcid.org/0000-0001-8827-8637

Chaim Putterman http://orcid.org/0000-0002-3204-6068

Tyler 0'Malley http://orcid.org/0000-0002-4439-1395

Thierry Dervieux http://orcid.org/0000-0002-3799-4438

\section{REFERENCES}

1 Hochberg MC. Updating the American College of rheumatology revised criteria for the classification of systemic lupus erythematosus. Arthritis Rheum 1997;40:1725.

2 Petri M, Orbai A-M, Alarcón GS, et al. Derivation and validation of the systemic lupus international collaborating clinics classification criteria for systemic lupus erythematosus. Arthritis Rheum 2012;64:2677-86.

3 Aringer M, Costenbader K, Daikh D, et al. 2019 European League against Rheumatism/American College of rheumatology classification criteria for systemic lupus erythematosus. Arthritis Rheumatol 2019;71:1400-12.

4 Manderson AP, Botto M, Walport MJ. The role of complement in the development of systemic lupus erythematosus. Annu Rev Immunol 2004;22:431-56.

5 Ramsey-Goldman R, Li J, Dervieux T, et al. Cell-Bound complement activation products in SLE. Lupus Sci Med 2017;4:e000236.

6 Kalunian KC, Chatham WW, Massarotti EM, et al. Measurement of cell-bound complement activation products enhances diagnostic performance in systemic lupus erythematosus. Arthritis Rheum 2012;64:4040-7.

7 Wallace DJ, Silverman SL, Conklin J, et al. Systemic lupus erythematosus and primary fibromyalgia can be distinguished by testing for cell-bound complement activation products. Lupus Sci Med 2016;3:e000127.

8 Putterman C, Furie R, Ramsey-Goldman R, et al. Cell-Bound complement activation products in systemic lupus erythematosus: comparison with anti-double-stranded DNA and standard complement measurements. Lupus Sci Med 2014;1:e000056.

9 Merrill JT, Petri MA, Buyon J, et al. Erythrocyte-bound C4d in combination with complement and autoantibody status for the monitoring of SLE. Lupus Sci Med 2018;5:e000263.

10 Bello GA, Brown MA, Kelly JA, et al. Development and validation of a simple lupus severity index using ACR criteria for classification of SLE. Lupus Sci Med 2016;3:e000136.

11 Dervieux T, Conklin J, Ligayon J-A, et al. Validation of a multi-analyte panel with cell-bound complement activation products for systemic lupus erythematosus. J Immunol Methods 2017;446:54-9.

12 Crosslin KL, Wiginton KL. The impact of race and ethnicity on disease severity in systemic lupus erythematosus. Ethn Dis 2009;19:301-7.

13 Lim SS, Bayakly AR, Helmick CG, et al. The incidence and prevalence of systemic lupus erythematosus, 2002-2004: the Georgia lupus registry. Arthritis Rheumatol 2014;66:357-68.

14 Somers EC, Marder W, Cagnoli P, et al. Population-Based incidence and prevalence of systemic lupus erythematosus: the Michigan lupus epidemiology and surveillance program. Arthritis Rheumatol 2014;66:369-78.

15 Katz JD, Senecal JL, Rivest C, et al. A simple severity of disease index for systemic lupus erythematosus. Lupus 1993;2:119-23. 